\title{
Shock wave interaction with a transcritical fuel droplet
}

\author{
Bradley Boyd ${ }^{* 1}$, Dorrin Jarrahbashi ${ }^{1}$ \\ 1J. Mike Walker '66 Mechanical Engineering Department, \\ Texas A\&M University, College Station, USA \\ *Corresponding author email: bradley.w.boyd@gmail.com
}

\begin{abstract}
Shock-droplet interactions occur in a spectrum of high-speed propulsion systems involving liquid fuels. When the combustion chamber pressure is above the critical pressure of the fuel, transcritical behaviour involving the transition from liquid-like to gas-like states is observed. Our understanding of multiphase-shock interaction is significantly less developed than its gasphase counterpart (i.e., shock-bubble interaction) and is particularly limited at transcritical conditions. We consider the interaction of a shockwave with a liquid $n$-dodecane droplet exposed to a nitrogen environment at a supercritical pressure. A fully-conservative diffuseinterface framework coupled with the Peng-Robinson equation of state is developed to accurately determine the state of the fluid as the shock propagates through the droplet. The shock-droplet interaction results show the development of interfacial instabilities and an axial jet.
\end{abstract}

\section{Keywords}

Shock-bubble interaction; Diffuse-interface methods; Transcritical Flow; Peng-Robinson Equation of State.

\section{Introduction}

The design of current and future energy conversion systems is shifting toward supercritical pressures to enable performance gain, lighter and more reliable systems for space [1-7], aviation [8-14], ground transportation [15-20], and power generation [21]. Understanding the vaporization, breakup, and combustion of the fuel droplets is critical when developing highpressure combustion devices [22-26]. Shock-droplet interactions occur in a spectrum of highspeed propulsion systems involving liquid fuels. Key applications include ramjets and scramjets [27, 28]. In applications such as high-speed diesel injection, shock waves may be induced and interact with the fuel spray [29].

Shock-bubble and shock-droplet interactions have been the subject of many studies over the past decades [30-32]. The seminal work of Haas and Strutevant [30] revealed very complex phenomena occurring during shock interaction with helium and R22 refrigerant bubble. Later, these cases were numerically modelled highlighting the feature of the shock-bubble interactions [31]. Since then, many additional cases have been considered, including the bubble composition of SF6 and Krypton [33-36]. However, these studies are all conducted at atmospheric pressure and room temperature where the fluids are in a gaseous state. Understanding the disintegration of droplets impacted by shocks at supercritical conditions is relevant to liquid-fuelled scramjet engines during low hypersonic, i.e., start-up operations, and will inform the mixing and combustion behaviour of liquid fuel sprays in a spectrum of supercritical and high-speed propulsion systems, particularly in hypersonic flights.

Although a better understanding of the sub- and supercritical conditions has been obtained by numerical and experimental efforts, there is still a critical gap in the knowledge on the transcritical conditions where the transition from liquid-like to gas-like behaviour occurs by crossing the pseudo-boiling line [37, 38]. At transcritical conditions, both subcritical (twophase) and supercritical (diffusion-controlled) behaviours might emerge [15, 20]. Classical 
transcritical cases involve fuel injected at a pressure above the critical pressure. In the case of fuel injection into a combustion chamber, the fuel with temperature initially below the critical temperature is injected at supercritical pressure, mixes with the hot ambient air, and increases the temperature of the fuel. As the fuel is heated, it transitions from a liquid-like to gas-like supercritical fluid and crosses the Nishikawa Widom line (termed pseudo-boiling). At a highertemperature, the fuel will behave as an ideal gas when the compressibility factor is equal to unity - i.e., $\mathrm{n}$-dodecane at $1100 \mathrm{~K}$ and $6 \mathrm{MPa}$. For the design of high-pressure and high-speed propulsion systems, it is of great interest to understand the behaviours of fuel droplets impacted by shockwave at pressures above the critical pressure. The lack of knowledge on multiphase shock-driven instabilities due to the dearth of detailed experimental data at such high pressure (temperature) is the main motivation behind this computational study that focuses on the shock interaction with fuel droplets at supercritical pressures.

There are many reports on simulation of droplet/shock interactions using sharp-interface and diffuse-interface approaches at subcritical conditions [39-44]. However, there are only two reports that simulate the fuel droplet-shock interaction at transcritical conditions $[45,46]$. To the authors' knowledge, the underlying physics behind this problem have not been previously investigated. In the study of our previous work [46], we briefly considered the shock-interaction with an $\mathrm{n}$-dodecane droplet in a nitrogen environment at near-critical conditions. It is important to note that the physics associated with a fluid near the critical point is significantly different from conventional liquid droplets or ideal-gas bubbles. For the shock-bubble interaction problem, the sphere of fluid is referred to as a bubble because it is in a gaseous state, typically an ideal gas. The term droplet is typically associated with a liquid sphere with significant surface tension effects. However, this differentiation breaks down for a supercritical fluid where gas-like and liquid-like properties better represent the state of the fluid [47]. Additionally, at supercritical conditions, surface tension is typically neglected. For this study, however, we use the term 'droplet' for the fuel sphere. In the present paper, we present the shock interaction with the supercritical fuel droplet by visualizing the shock characteristics and instability development.

\section{Methodology}

We consider a fully compressible (FC), inviscid, multiphase model. The governing equations for a diffuse-interface two-species system are given below:

$$
\begin{gathered}
\frac{\partial(\rho)}{\partial t}+\nabla \cdot(\rho \mathbf{u})=0 \\
\frac{\partial\left(\rho Y_{D}\right)}{\partial t}+\nabla \cdot\left(\rho \mathbf{u} Y_{D}\right)=0 \\
\frac{\partial(\rho \mathbf{u})}{\partial t}+\nabla \cdot(\rho \mathbf{u} \otimes \mathbf{u}+p \mathbf{I})=\mathbf{0} \\
\frac{\partial E}{\partial t}+\nabla \cdot(\mathbf{u}(E+p))=0,
\end{gathered}
$$

where $Y_{D}$ is the mass fraction of the bubble/droplet fluid species, $\rho$ is the density, $\mathbf{u}$ is the velocity vector, $p$ is the pressure, $E$ is the total energy $E=\rho\left(e+|\mathbf{u}|^{2} / 2\right), e$ is the internal energy, and $\mathbf{I}$ is the identity matrix. The mass fraction of the second species of the twocomponent system is given by the mixture rule $Y_{S}=1-Y_{D}$. The system of equations, Eqs. (1) -(4), is closed using the PR-EoS [48] as follows

$$
p=\frac{R_{U} T}{V_{m}-b}-\frac{a}{V_{m}^{2}+2 b V_{m}-b^{2}},
$$


where $T$ is the temperature, $R_{U}$ is the universal gas constant, $V_{m}$ is the molar volume $V_{m}=M / \rho, M$ is the molar mass, and $a$ and $b$ are coefficients that depend on the state and composition of the fluid. The PR-EoS is modified [46] to approximate the state of the fluid in the vapor dome region to increase the robustness of the numerical method. The parameters for the NASA polynomials that are used to determine the internal energy, enthalpy, and entropy were taken from Ref [49]

This study uses an in-house, density-based, finite volume solver in $\mathrm{C}++$ parallelized using domain decomposition and message passing interface (MPI) which is presented in [46]. In the same study, we introduced a hybrid (HY) numerical method, which hybridizes the FC procedure with the DF method [46]. HY method is chosen for this study as our earlier study proved, the HY method can significantly reduce the magnitude of pressure oscillation while limiting the loss of energy conservation when a shock impacts the nitrogen-n-dodecane interface. To avoid the interaction of the characteristic fields and increase the robustness and stability of the solver, we reconstruct the primitive variables in the characteristic space Characteristic-wise reconstruction (CW) [46, 50-53].

The temporal discretization of Eqs. (1)-(4) uses the third-order total-variation-diminishing Runge-Kutta scheme (RK3-TVD) [54]. The Godunov flux is determined using the HartenLax-van Leer-contact (HLLC) approximate Riemann flux [50, 55, 56]. Additionally, we make use of a maximum-principle-satisfying and positivity-preserving flux limiter to help ensure the boundness of the mass fraction and the positivity of density and pressure [55-57].

WENO5-CW reconstruction is performed in the axisymmetric cylindrical coordinates $-r, z$ (see Appendix $\mathrm{C}$ of [58] for more details). We also apply the artificial interface thickening method as discussed in our earlier study [46] to the initial condition of the droplet interface in axisymmetric cylindrical coordinates with $\beta=2$, where $\beta$ is the number of finite volume cells the interface is initially smeared over. The interface thickening reduces the jump in properties between cells, reducing pressure oscillations and increasing the numerical stability [46]. Additionally, interface thickening reduces the loss in energy conservation when used with a quasi-conservative method like the HY method [46]. Because the droplet is defined on a uniform Cartesian mesh, this interfacial thickening also helps provide the smooth curvature of the initial droplet shape.

\section{Results and Discussion}

We consider the interaction of a shock wave in a nitrogen environment with a droplet of ndodecane at supercritical pressure in a liquid-like state to showcase the transcritical shock interaction in 2D axisymmetric cylindrical coordinates. In this study, we focus on the ndodecane/nitrogen interface as n-dodecane has been extensively used as a diesel fuel surrogate in the combustion community.

The shock wave travels from right to left impacting the droplet, where the droplet diameter is $5 \mathrm{~cm}$, see Figure 1. The computational domain is shown in Figure 1 where the axial length is $50 \mathrm{~cm}$ (from $\mathrm{z}=-25$ to $\mathrm{z}=25 \mathrm{~cm}$ ) and with a radius of $30 \mathrm{~cm}$ (which is approximately $6 D_{0}$ as is the suggested dimension in Ref. [59]). The size of the computational domain is sufficiently large so that potential erroneous reflections from the non-reflective boundaries will not interact with the droplet throughout the simulation. The computational domain consists of a uniform mesh from $z=-16$ to $16 \mathrm{~cm}$ and from the axis of symmetry to $r=5 \mathrm{~cm}$ to increase the computational speed of the simulation where the cell dimensions are $\Delta z \approx \Delta r \approx 0.23 \mathrm{~mm}$. The mesh then grows at a rate of $10 \%$ in the z-direction to the outer boundaries. Due to the axis of symmetry boundary condition, only the top half of the depicted computational domain is 
required for the simulation (Figure 1). A transmissive BC is used at the other boundaries (left, right, and outer boundaries).

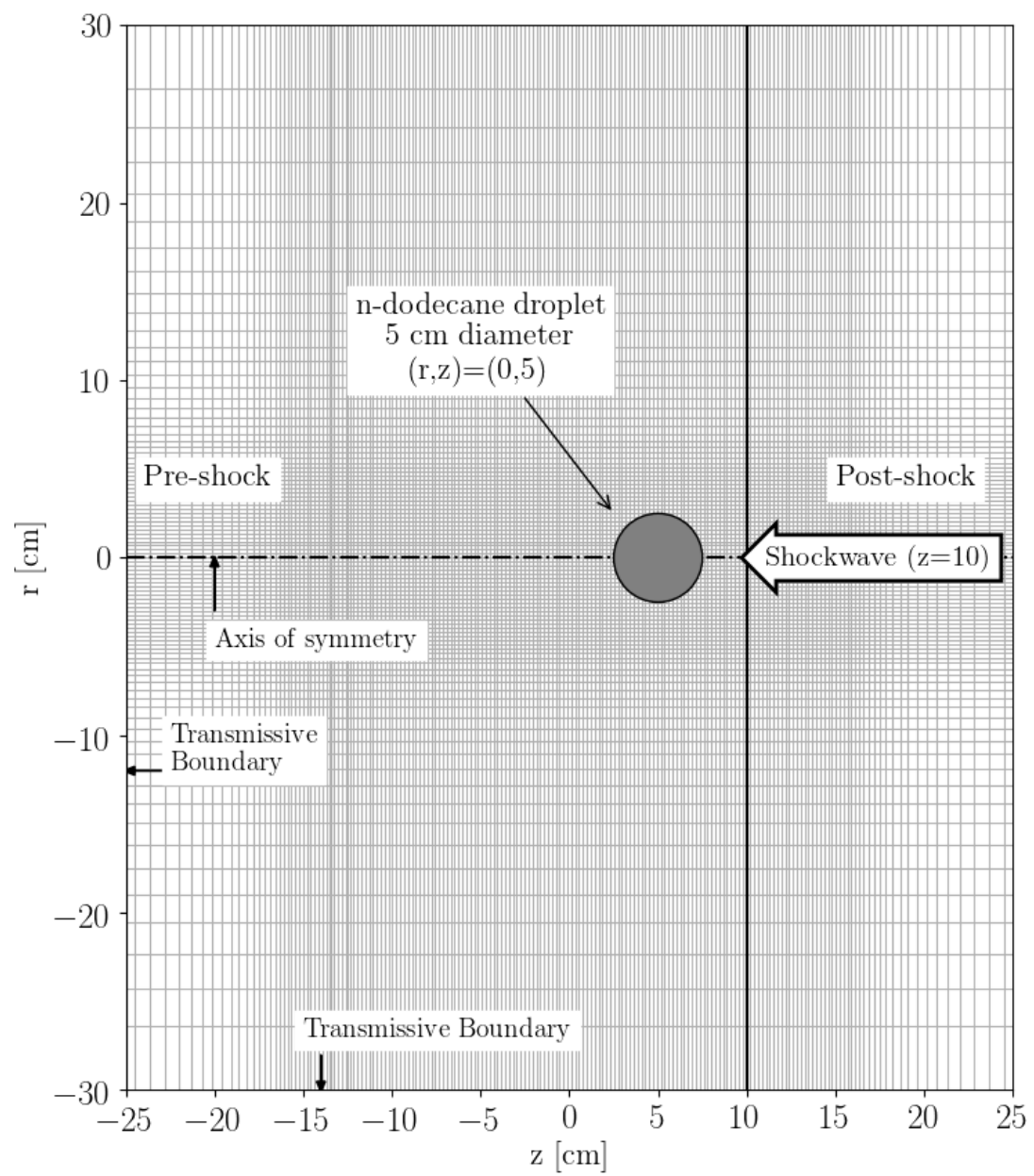

Figure 1. Depiction of the shock-droplet case. The depicted cell size is ten times larger than the cell size used in the simulations ( $\Delta z \approx \Delta r \approx 0.23 \mathrm{~mm}$ ). Due to the axis of symmetry boundary condition, only the top half of the depicted computational domain is required for the simulation.

Here we show the key flow features of the shock interaction with a fuel droplet where the droplet is initially at a pressure of $6 \mathrm{MPa}$ and a temperature is $650 \mathrm{~K}$. The critical temperature of $\mathrm{n}$-dodecane is $658.1 \mathrm{~K}$ and the critical pressure is $1.82 \mathrm{MPa}$. In this case, the $\mathrm{n}$-dodecane is at a supercritical pressure near the pseudo-boiling line (close to the critical temperature). For a Mach 1.2 shock wave, the initial state of the three regions, (1) pre-shock, (2) post-shock, and (3) n-dodecane droplet, are summarized below:

$\left(Y_{D}, u_{z}[\mathrm{~m} / \mathrm{s}], p[\mathrm{MPa}], T[\mathrm{~K}], \rho\left[\mathrm{kg} / \mathrm{m}^{3}\right]\right)=\left\{\begin{array}{cl}(0,0,6,650,30.46) & \text { pre-shock } \\ (0,-160.3,9.096,736.2,40.38) & \text { post-shock } \\ (1,0,6,650,419.9) & \text { droplet }\end{array}\right.$

Note that $u_{z}$ is the velocity component in the $z$-direction and the initial velocity in the $r$-direction $\left(u_{r}\right)$ is zero everywhere. The shock wave, which is initially at $z=10 \mathrm{~cm}$, travels to the left towards the droplet and $t$ begins when the shock first impacts the droplet interface (at $z=7.5$ $\mathrm{cm})$. 
Once the shock impacts the droplet, the vertical incident shock above and below the droplet is joined by two shock waves: (1) the refracted shock that lies within the droplet and (2) the reflected shock that is outside the droplet (Figure 2(a)). As time progresses, the refracted shock travels through the undisturbed region and converges towards the downstream interface (Figure 2(b)). The passage of the refracted shock across the downstream interface results in a transmitted shock and the reflection of a rarefaction wave, Figure 2(c). The acoustic impedance mismatch experienced by the refracted shock at the downstream interface results in the internal reflection of a rarefaction wave [32]. As time progresses, interfacial instabilities begin to grow at the interface between the fuel and the surrounding nitrogen, Figure 2(d). After the shock-droplet interaction, an axial jet develops outwards at the downstream interface, Figure 2(c)-(d).

Mach 1.2 shock/droplet interaction at $650 \mathrm{~K}$ and $6 \mathrm{MPa}$
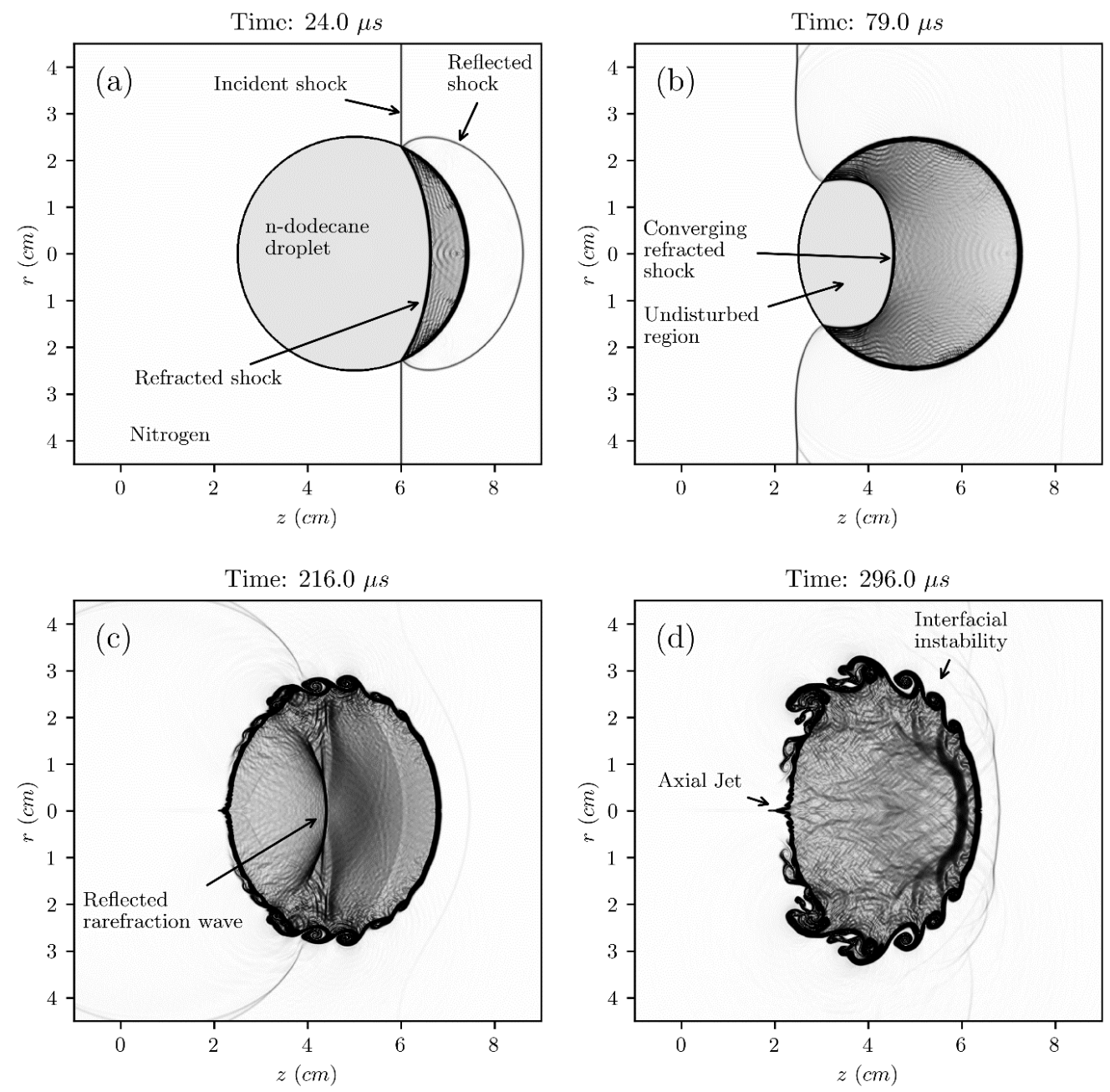

Figure 2. Numerical schlieren images for the Mach 1.2 shock/droplet interaction after (a) $24 \mu \mathrm{s}$, (b) $76 \mu \mathrm{s}$, (c) 216 $\mu \mathrm{s}$, and (d) $296 \mu \mathrm{s}$.

The axial jet is a result of the refracted shock wave convergence (Figure 3 ). The refracted shock convergence reaches a focal point just inside the droplet near the downstream interface. Figure 3(a) shows the point in time where the refracted shock has fully wrapped around the undisturbed region and is near the focal point. Figure $3(\mathrm{~b})$ shows the axial pressure distribution before shock convergence, where the leftward traveling shock has a significantly higher pressure of about $18 \mathrm{MPa}$. There is no more undisturbed region once the shock converges (Figure 3(c)) and the peak pressure at the axis near the downstream interface reaches about 
$30 \mathrm{MPa}$ (Figure 3(d)). After the shock convergence, the shock wave is reflected as a strong rarefaction wave as seen in Figure $3(\mathrm{e})-(\mathrm{f})$ where the pressure drop is large (about $5 \mathrm{MPa}$ ).
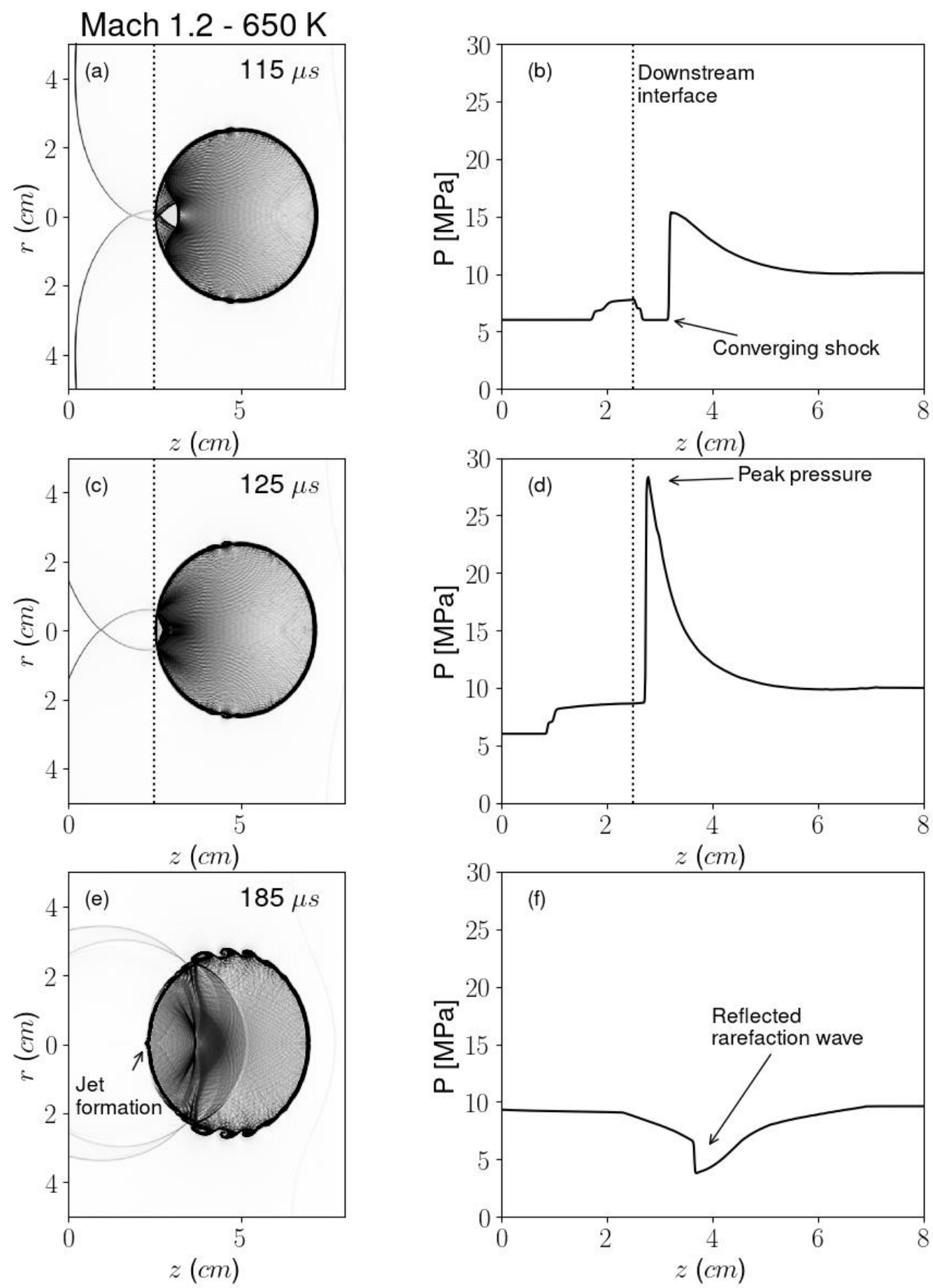

Figure 3. The numerical schlieren images ((a), (c), (e)) and the corresponding axial pressure distributions ((b), (d), (f)) show the shock convergence resulting in a localized high-pressure region inside the downstream droplet interface. The pressure peak in (d) causes the formation of an axial jet.

\section{Conclusions}

In this paper, we developed a model to simulate the shock wave interaction with a fuel droplet at supercritical pressure. The shock-droplet resulted in various interesting flow features, including axial jetting and interfacial instability. The model developed will be used in future studies to further investigate the physics of the transcritical shock-droplet interaction. 


\section{Acknowledgments}

Portions of this research were conducted with the advanced computing resources provided by Texas A\&M High-Performance Research Computing.

\section{References}

1. Hersch, M. and E.J. Rice, Gaseous-hydrogen liquid-oxygen rocket combustion at supercritical chamber pressures. 1967.

2. Oefelein, J.C., Thermophysical characteristics of shear-coaxial LOX-H2 flames at supercritical pressure. Proceedings of the Combustion Institute, 2005. 30(2): p. 2929-2937.

3. $\quad$ Harrje, D.T., Liquid propellant rocket combustion instability. 1972.

4. Okong', N., K. Harstad, and J. Bellan, Direct numerical simulations of $\mathrm{O} / \mathrm{H}$ temporal mixing layers under supercritical conditions. AIAA journal, 2002. 40(5): p. 914-926.

5. Miller, R.S., K.G. Harstad, and J. Bellan, Direct numerical simulations of supercritical fluid mixing layers applied to heptane-nitrogen. Journal of Fluid Mechanics, 2001. 436: p. 1-39.

6. Miller, R., K. Harstad, and J. Bellan, Evaluation of equilibrium and non-equilibrium evaporation models for many-droplet gas-liquid flow simulations. International Journal of Multiphase Flow, 1998. 24(6): p. 10251055.

7. Bellan, J., Supercritical (and subcritical) fluid behavior and modeling: drops, streams, shear and mixing layers, jets and sprays. Progress in Energy and Combustion Science, 2000. 26(4): p. 329-366.

8. H. Mayer, W.O., et al., Atomization and breakup of cryogenic propellants under high-pressure subcritical and supercritical conditions. Journal of Propulsion and Power, 1998. 14(5): p. 835-842.

9. Zong, N. and V. YANG*, Cryogenic fluid jets and mixing layers in transcritical and supercritical environments. Combustion science and technology, 2006. 178(1-3): p. 193-227.

10. Chehroudi, B., D. Talley, and E. Coy, Visual characteristics and initial growth rates of round cryogenic jets at subcritical and supercritical pressures. Physics of Fluids, 2002. 14(2): p. 850-861.

11. Davis, D.W., On the behavior of a shear-coaxial jet, spanning sub-to supercritical pressures, with and without an externally imposed transverse acoustic field. 2006, PENNSYLVANIA STATE UNIV UNIVERSITY PARK DEPT OF MECHANICAL AND NUCLEAR ....

12. Chehroudi, B.J.I.J.o.A.E., Recent experimental efforts on high-pressure supercritical injection for liquid rockets and their implications. 2012. 2012.

13. Leyva, I., B. Chehroudi, and D. Talley. Dark core analysis of coaxial injectors at sub-, near-, and supercritical conditions in a transverse acoustic field. in 43rd AIAA/ASME/SAE/ASEE Joint Propulsion Conference \& Exhibit. 2007.

14. Chehroudi, B., Physical Hypothesis for the Combustion Instability in Cryogenic Liquid Rocket Engines. Journal of Propulsion and Power, 2010. 26(6): p. 1153-1160.

15. Oefelein, J., et al., Effects of Real-Fluid Thermodynamics on High-Pressure Fuel Injection Processes. SAE Int. J. Engines, 2014. 7(3): p. 1125-1136.

16. Dahms, R.N., J.C.J.C. Oefelein, and Flame, Liquid jet breakup regimes at supercritical pressures. 2015. 162(10): p. 3648-3657.

17. Dahms, R.N. and J.C. Oefelein, On the transition between two-phase and single-phase interface dynamics in multicomponent fluids at supercritical pressures. Physics of Fluids, 2013. 25(9): p. 092103.

18. Dahms, R.N. and J.C. Oefelein, Non-equilibrium gas-liquid interface dynamics in high-pressure liquid injection systems. Proceedings of the Combustion Institute, 2015. 35(2): p. 1587-1594.

19. Dahms, R.N., et al., Understanding high-pressure gas-liquid interface phenomena in Diesel engines. Proceedings of the Combustion Institute, 2013. 34(1): p. 1667-1675.

20. Dahms, R.N., Understanding the breakdown of classic two-phase theory and spray atomization at enginerelevant conditions. Physics of Fluids, 2016. 28(4): p. 042108.

21. Lettieri, C., G. Subashki, and Z. Spakovszky. Modeling Near Critical and Supercritical Fuel Injection and Mixing in Gas Turbine Applications. in ASME Turbo Expo 2018: Turbomachinery Technical Conference and Exposition. 2018. American Society of Mechanical Engineers.

22. Aggarwal, S.K., C. Yan, and G. Zhu, Transcritical vaporization of a liquid fuel droplet in a supercritical ambient. Combustion Science and Technology, 2002. 174(9): p. 103-130.

23. Gao, Y., et al., Breakup, instabilities, and dynamics of high-speed droplet under transcritical conditions. Advances in Mechanical Engineering, 2015. 7(6): p. 1687814015588469.

24. Yang, S., et al., Evaporation and dynamic characteristics of a high-speed droplet under transcritical conditions. Advances in Mechanical Engineering, 2016. 8(4): p. 1687814016642953.

25. Yang, V., Modeling of supercritical vaporization, mixing, and combustion processes in liquid-fueled propulsion systems. Proceedings of the Combustion Institute, 2000. 28(1): p. 925-942.

26. Yang, V., N. N, and J.-S. Shuen, Vaporization of Liquid Oxygen (LOX) Droplets in Supercritical Hydrogen Environments. Combustion Science and Technology, 1994. 97(4-6): p. 247-270.

27. Ren, Z., et al., Numerical studies on supersonic spray combustion in high-temperature shear flows in a scramjet combustor. Chinese Journal of Aeronautics, 2018. 31(9): p. 1870-1879.

28. Ren, Z., et al., Supersonic spray combustion subject to scramjets: Progress and challenges. Progress in Aerospace Sciences, 2019. 105: p. 40-59. 
29. Jia, T.-M., Y.-S. Yu, and G.-X. Li, Experimental investigation of effects of super high injection pressure on diesel spray and induced shock waves characteristics. Experimental Thermal and Fluid Science, 2017. 85: p. 399-408.

30. Haas, J.-F. and B. Sturtevant, Interaction of weak shock waves with cylindrical and spherical gas inhomogeneities. Journal of Fluid Mechanics, 1987. 181: p. 41-76.

31. Quirk, J.J. and S. Karni, On the dynamics of a shock-bubble interaction. Journal of Fluid Mechanics, 1996. 318: p. 129-163.

32. Ranjan, D., J. Oakley, and R. Bonazza, Shock-bubble interactions. Annual Review of Fluid Mechanics, 2011. 43: p. 117-140.

33. Layes, G., G. Jourdan, and L. Houas, Distortion of a spherical gaseous interface accelerated by a plane shock wave. Physical review letters, 2003. 91(17): p. 174502.

34. Niederhaus, J.H.J., A computational parameter study for three-dimensional shock-bubble interactions. 2007.

35. Zhai, Z.-G., et al., Jet formation in shock-heavy gas bubble interaction. Acta Mechanica Sinica, 2013. 29(1): p. 24-35.

36. Zhu, Y., et al., Jet formation of SF6 bubble induced by incident and reflected shock waves. Physics of Fluids, 2017. 29(12): p. 126105.

37. Banuti, D., et al., Seven questions about supercritical fluids - towards a new fluid state diagram, in 55th AIAA Aerospace Sciences Meeting.

38. Raju, M., et al., Widom Lines in Binary Mixtures of Supercritical Fluids. Scientific Reports, 2017. 7(1): p. 3027.

39. Das, P. and H.S. Udaykumar, A sharp-interface method for the simulation of shock-induced vaporization of droplets. Journal of Computational Physics, 2020. 405(109005).

40. Daniel P. Garrick, M.O., Jonathan D. Regele, A finite-volume HLLC-based scheme for compressible interfacial flows with surface tension. Journal of Computational Physics, 2017. 339: p. 46-67

41. Daniel P. Garrick, W.A.H., Jonathan D. Regele, An interface capturing scheme for modeling atomization incompressible flows. Journal of Computational Physics 2017. 344: p. 260-280

42. J. C. Meng, T.C., Numerical simulations of the early stages of high-speed droplet breakup. Shock Waves, 2014. 24 (6).

43. Jomela C. Meng, T.C., Numerical simulation of the aerobreakup of a water droplet. Journal of Fluid Mechanics, 2018. 835: p. 1108-1135

44. Q. Wan, H.J., R. Deiterding, V. Eliasson, Numerical and experimental investigation of oblique shock wave refection of a water wedge. Jounal of Fluid Mechanics, 2017. 826: p. 732-758.

45. Tudisco, P. and S. Menon, Numerical Investigations of Phase-Separation During Multi-Component Mixing at Super-Critical Conditions. Flow, Turbulence and Combustion, 2020. 104(2): p. 693-724.

46. Boyd, B. and D. Jarrahbashi, A diffuse-interface method for reducing spurious pressure oscillations in multicomponent transcritical flow simulations. Computers \& Fluids, 2021: p. 104924.

47. Abgrall, R., How to Prevent Pressure Oscillations in Multicomponent Flow Calculations: A Quasi Conservative Approach. Journal of Computational Physics, 1996. 125(1): p. 150-160.

48. Peng, D.-Y. and D.B. Robinson, A new two-constant equation of state. Industrial \& Engineering Chemistry Fundamentals, 1976. 15(1): p. 59-64.

49. McBride, B.J., Coefficients for calculating thermodynamic and transport properties of individual species. Vol. 4513. 1993: NASA Langley Research Center.

50. Johnsen, E. and T. Colonius, Implementation of WENO schemes in compressible multicomponent flow problems. Journal of Computational Physics, 2006. 219(2): p. 715-732.

51. Coralic, V. and T. Colonius, Finite-volume WENO scheme for viscous compressible multicomponent flows. Journal of computational physics, 2014. 274: p. 95-121.

52. Harten, A., et al., Uniformly High Order Accurate Essentially Non-oscillatory Schemes, III. Journal of Computational Physics, 1997. 131(1): p. 3-47.

53. Qiu, J. and C.-W. Shu, On the Construction, Comparison, and Local Characteristic Decomposition for High-Order Central WENO Schemes. Journal of Computational Physics, 2002. 183(1): p. 187-209.

54. Gottlieb, S. and C.-W. Shu, Total variation diminishing Runge-Kutta schemes. Mathematics of computation, 1998. 67(221): p. 73-85.

55. Boyd, B. and S. Becker, Numerical modelling of an acoustically-driven bubble collapse near a solid boundary. Fluid Dynamics Research, 2018. 50(6): p. 065506.

56. Boyd, B. and S. Becker, Numerical modeling of the acoustically driven growth and collapse of a cavitation bubble near a wall. Physics of Fluids, 2019. 31(3): p. 032102.

57. Zhang, X. and C.-W. Shu, Maximum-principle-satisfying and positivity-preserving high-order schemes for conservation laws: survey and new developments. Proceedings of the Royal Society A: Mathematical, Physical and Engineering Sciences, 2011. 467(2134): p. 2752-2776.

58. Boyd, B., Numerical modelling of an acoustically-driven bubble: the growth and collapse near a wall. 2018.

59. Meng, J.C. and T. Colonius, Numerical simulation of the aerobreakup of a water droplet. Journal of Fluid Mechanics, 2018. 835: p. 1108. 